\title{
Central precocious puberty due to hypothalamic hamartoma in neurofibromatosis type 1
}

\author{
Emanuele Bartolini, ${ }^{1,2}$ Stefano Stagi, ${ }^{3}$ Perla Scalini, ${ }^{3}$ Andrea Bianchi, ${ }^{4}$ \\ Antonio Ciccarone, ${ }^{5}$ Mario Mascalchi ${ }^{4}$
}

\begin{abstract}
${ }^{1}$ Neurology Unit and Laboratories, Anna Meyer Children's University Hospital, Florence; ${ }^{2}$ Stella Maris Foundation, Calambrone, Pisa; ${ }^{3}$ Health Sciences Department, University of Florence, Anna Meyer Children's University Hospital, Florence; ${ }^{4}$ Quantitative and Functional Neuroradiology Research Program at Anna Meyer Children's and Careggi University Hospitals, University of Florence, Florence; ${ }^{5}$ Medical Physics Unit at Anna Meyer Children's University Hospital, Florence; Italy
\end{abstract}

\section{Dear Editor,}

In neurofibromatosis type 1 (NF1), Central Precocious Puberty (CPP) occurs almost invariably in association with optic pathway tumors, usually pilocytic astrocytoma. ${ }^{1}$ However, at times the aetiology remains undefined. ${ }^{2}$ On the other hand, in the general population CPP develops due to an underlying hypothalamic hamartoma in up to $10 \%$ of patients. ${ }^{3} \mathrm{To}$ the best of our knowledge, CPP due to hypothalamic hamartomas in NF1 has been reported only once in the literature. ${ }^{4}$

Herein we report the case of a young girl with NF1 who developed CCP related to a hamartoma of the tuber cinereum at 5 years of age. NF1 was diagnosed at 3 months of age because of the presence of

Key words: Brain MRI, Diagnosis, Neuroimaging, Spectroscopy, Therapy

\footnotetext{
Address for correspondence:

Emanuele Bartolini, MD, Pediatric Neurology Unit and Laboratories, Anna Meyer Children's Hospital, Viale Pieraccini 24, 50139, Florence, Italy; Tel.: +390555662573;

Fax: +390555662329, E-mail: emanuele.bartolini@meyer.it

Received: 19-10-2015, Accepted: 21-10-15
}

about 30 café-au-lait cutaneous spots (diameter $>0.5$ $\mathrm{mm}$ ) associated with iris Lisch nodules. Thereafter she developed axillary and groin freckling and three subcutaneous neurofibromas. Familial clinical history was negative. Precocious puberty was diagnosed at age 5.3 years, with increased growth velocity (from the $25^{\text {th }}$ to the $90^{\text {th }}$ percentile) and thelarche (Tanner breast stage 2). LH-RH test was positive (luteinizing hormone -LH- peak $10.1 \mathrm{mUI} / \mathrm{ml}$ ). Bone age was correspondent to chronological age, while pelvic ultrasonography revealed increased ovarian volume and a transitional uterus. Brain Magnetic Resonance Imaging (MRI) showed an oval 13x14 mm mass in the tuber cinereum region, isointense to gray matter on all sequences, with no displacement on the third ventricle and no contrast enhancement, consistent with hypothalamic hamartoma. ${ }^{5}$ In addition, multiple round areas of $\mathrm{T} 2$ hyperintensity consistent with unidentified bright objects (UBOs) and not showing contrast enhancement were present in the peridentate white matter, right superior cerebellar peduncle and hippocampus, bilaterally. Treatment with intramuscular Enantone $3.5 \mathrm{mg}$ every 28 days was started with a rapid biochemical and clinical response (after 6 months of therapy, Tanner breast stage was 1 , LH peak was $1.4 \mathrm{mUI} / \mathrm{ml})$. Treatment was suspended at the age of 11.3 years. During follow-up the pituitary function was always normal. No seizure occurred, 
most importantly of the gelastic type. Serial control MRIs were performed until age 18 and demonstrated no changes of the tuber cinereum lesion (Figure 1) or UBOs. At the last 3T MRI we also performed quantitative proton MRI spectroscopy using an external reference phantom. ${ }^{6}$ Single voxel short TE spectra were obtained from the hypothalamic lesion (Figure 1) and from the apparently normal right parietal white matter: this showed a lower concentration $(8.4 \mathrm{mmol} / \mathrm{kg})$ of $\mathrm{N}$-acetyl-aspartate (NAA) and a higher concentration $(4.6 \mathrm{mmol} / \mathrm{kg})$ of myo-inositol $(\mathrm{mI})$ and choline (Cho) $(3.0 \mathrm{mmol} / \mathrm{kg})$ in the former compared to the latter (NAA $13.7 \mathrm{mmol} / \mathrm{kg}$; $\mathrm{mI} 1.6 \mathrm{mmol} / \mathrm{kg}$; Cho 1.6 $\mathrm{mmol} / \mathrm{kg}$ ), whereas the concentration of creatine was similar (lesion $9.9 \mathrm{mmol} / \mathrm{kg}$; normal WM $10.3 \mathrm{mmol} /$ $\mathrm{kg}$ ). These quantitative data are in agreement with the typical spectroscopy pattern of a tuber cinereum

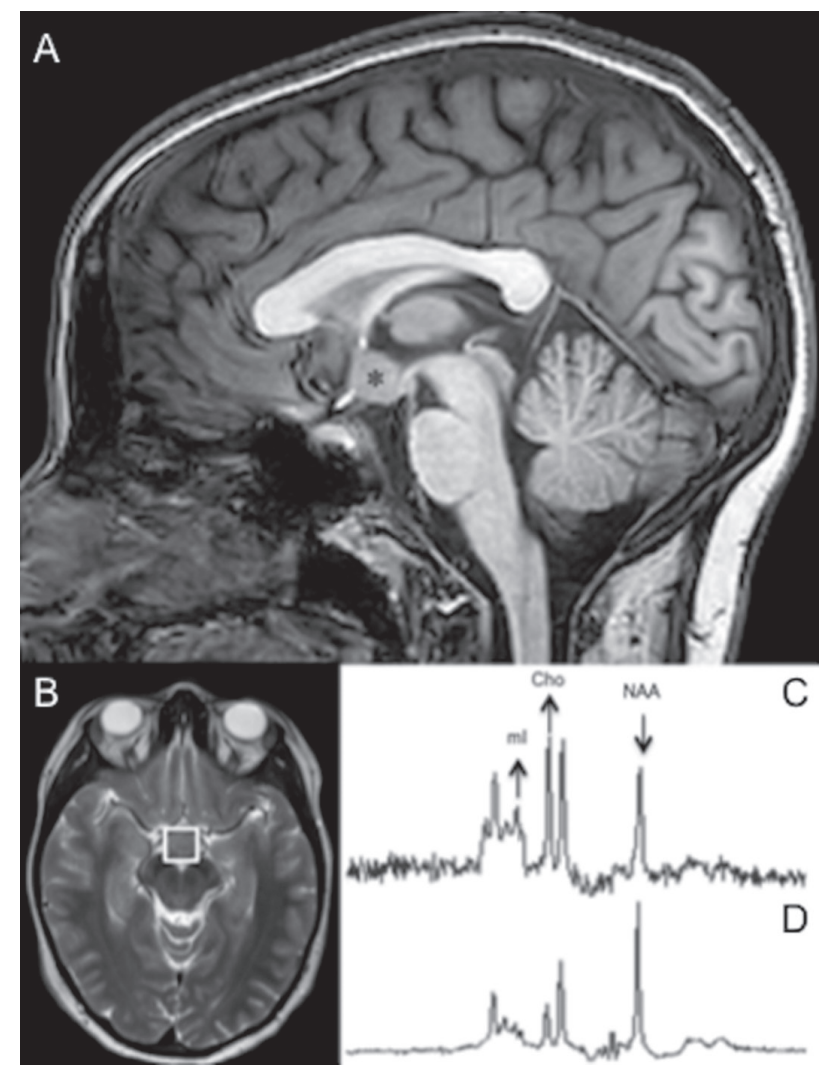

Figure 1. Sagittal T1 weighted image (A) shows a midline formation (*) isointense to the GM in the region of the tuber cinereum. Axial T2 weighted image (B) showing single voxel used for proton MR spectroscopy (PRESS TR 2000ms; TE $53 \mathrm{~ms}$ ) of the lesion. The lesion spectrum $(\mathrm{C})$ demonstrates low NAA and high Cho and $\mathrm{mI}$ as compared to normal parietal WM spectrum (D). hamartoma. ${ }^{5}$ In particular, increased myo-inositol is assumed to reflect increased glial content, whereas decreased NAA is related to neuronal substitution.

Hypothalamic hamartomas are heterotopic nonneoplastic masses of grey matter composed of neurons and glia cells of normal appearance and structure. ${ }^{5}$ They are usually small lesions, measuring between 0.5 and $2 \mathrm{~cm}$ in diameter, which as a rule remain unchanged over time. The typical location is in the midline, in proximity to the tuber cinereum and the mammillary bodies.

From the neurobiological point of view, hamartomas are to be distinguished from UBOs which are common in NF1 and typically arise before adolescence and disappear in adult age. UBOs are unlikely to be related to CPP because they are usually localized symmetrically or asymmetrically in the cerebral hemispheres, brainstem and cerebellum. It should be noted, however, that differential diagnosis between hypothalamic hamartoma and optic pathway glioma on MRI and proton MR spectroscopy is based on signal heterogeneity and frequent presence of contrast enhancement areas, as well of lactate and lipids in the MR spectroscopy of optic pathway gliomas. ${ }^{7}$

In conclusion, our observations suggest that $\mathrm{CPP}$ can be due to a tuber cinereum hamartoma also in NF1, with diagnostic, prognostic and therapeutic implications that appear analogous to hamartoma-related CPP in the general population. Conversely, the admittedly rare possibility of NF1 has to be entertained in every new diagnosis of hamartoma-related CPP.

\section{DISCLOSURE}

The authors report no disclosures relevant to the manuscript.

\section{REFERENCES}

1. Virdis R, Sigorini M, Laiolo A et al, 2000 Neurofibromatosis type 1 and precocious puberty. J Pediatr Endocrinol Metab 13: Suppl 1: 841-844.

2. Bizzarri C, Bottaro G, 2015 Endocrine implications of neurofibromatosis 1 in childhood. Horm Res Paediatr 83: 232-241.

3. Ng SM, Kumar Y, Cody D, Smith CS, Didi M, 2003 Cranial MRI scans are indicated in all girls with central precocious puberty. Arch Dis Child 88: 414-418. 
4. Biswas K, Kapoor A, Jain S, Ammini AC, 2000 Hypothalamic hamartoma as a cause of precocious puberty in neurofibromatosis type 1: patient report. J Pediatr Endocrinol Metab 13: 443-444.

5. Amstutz DR, Coons SW, Kerrigan JF, Rekate HL, Heiserman JE, 2006 Hypothalamic hamartomas: Correlation of MR imaging and spectroscopic findings with tumor glial content. AJNR 27: 794-798.
6. Guerrini L, Belli G, Mazzoni L, et al, 2009 Impact of cerebrospinal fluid contamination on brain metabolites evaluation with $1 \mathrm{H}-\mathrm{MR}$ spectroscopy: a single voxel study of the cerebellar vermis in patients with degenerative ataxias. J Magn Reson Imaging 30: 11-17.

7. Barkovich AJ Moore KR, Jones BV, et al 2007. In Diagnostic imaging: pediatric neuroradiology. First Ed. Elsevier (eds); pp, I2:14-16. 